\title{
Reactive Power Planning and Its Cost Allocation for Distribution Systems with Distributed Generation
}

\author{
Lin Chen, Jin Zhong, Member, IEEE, Deqiang Gan
}

\begin{abstract}
This paper addresses some of the modeling and economic issues pertaining to the optimal reactive power planning of radial distribution systems with distributed generation. When wind power generation (WPG) units are installed in a distribution system, they may cause reverse power flows and voltage variations due to the random-like outputs of wind turbines. To solve this problem, we introduce Static Var Compensator (SVC) into distribution systems, and combine the reactive power support from distributed diesel units for voltage control. An optimal reactive power planning model is proposed in this paper. Monte-Carlo simulation is used to simulate the uncertainty of wind power generation. The locations and the outputs of SVCs and distributed diesel units are determined using our proposed optimal reactive power planning model. Genetic Algorithm (GA) is used to solve the optimization problem. Furthermore, we apply the Shapley Value Axiom in cooperative game theory to allocate the reactive power cost of SVC among wind power turbines, which have caused voltage variations. Finally, we discuss the allocation results from an economic point of view.
\end{abstract}

Index Terms-Distributed Generation, SVC, Monte-Carlo simulation, optimal allocation, cost allocation

\section{NOMENCLATURE}

$t \quad$ Wind power generation units output status;

$T_{t} \quad$ The time duration of status $t$;

$K \quad$ The energy cost per unit;

$P_{t}(x)$ Active power loss for wind power output status $t$;

$i \quad$ System node;

$e_{i} \quad$ Binary variable, the value depends on whether SVC is installed at bus $i$ or not;

$r_{i} \quad$ Marginal cost of SVC at bus $i$;

$Q_{c i}^{0} \quad$ The maximum required capacity of SVC placed at bus $i$ for all the WPG output statuses;

This work was supported by the Research Grand Council of Hong Kong under Grant HKU7201/05E and HKU Seed Funding Program for Basic Research (project code: 200411159086).

L. Chen and D. Gan are with the College of Electrical Engineering, Zhejiang University, Hangzhou, China (e-mail: chenlinzju@163.com, dgan@zju.edu.cn)

J. Zhong is with the Department of Electrical and Electronic Engineering, The University of Hong Kong, Pokfulam Road, Hong Kong. (e-mail: jzhong@eee.hku.hk). $c_{i} \quad$ Fixed installation cost of SVC at bus $i$;

$P_{D G i}^{t}, Q_{D G i}^{t}$ Diesel unit active and reactive power outputs at node $i$ for WPG output status $t$;

$a_{i}, b_{i}, c_{i}$ The coefficients of production cost function for the diesel unit at node $i$;

$e_{i W P G} \quad$ Binary variable, the value depends on whether wind turbine is installed at bus $i$ or not;

$e_{i D G} \quad$ Binary variable, the value depends on whether diesel unit is installed at bus $i$ or not;

$P_{W P G i}^{t}, Q_{W P G i}^{t}$ The active and reactive power outputs of the wind power generator at node $i$ for status $t$;

$P_{D G i}^{t}, Q_{D G i}^{t}$ The active and reactive power outputs of the diesel unit at node $i$ for status $t$;

$V_{i}^{t} \quad$ Voltage magnitude at node $i$ for WPG output status $t$;

$Q_{c i}^{t} \quad$ Reactive power injection by SVC at node $i$ for WPG output status $t$;

$Q_{c i}^{\max } \quad$ The maximum reactive power output of the SVC at node $i$

$P_{D G i}^{\max }, Q_{D G i}^{\max }$ The maximum active and reactive power outputs of the diesel unit at node $i$.

\section{INTRODUCTION}

$\mathrm{W}$ ITH the deregulation of electric power systems and the development of new generation technologies, distributed generation (DG) is becoming more and more important in the future power systems. One of the benefits of DG sources is deferring or avoiding transmission and distribution expansions. In general, DG can be defined as small-scale electric power generation sources (roughly $30 \mathrm{MW}$ or less). They are usually connected to distribution networks or located at the customer side. The distributed generation sources include those generators with traditional power technologies, such as, diesel and combustion turbines, and power sources of renewable technologies, such as, photovoltaic and wind power.

Wind power generation has become one of the most 
commonly used renewable energy sources. Diesel generation is widely used in distribution systems and can be used as a reactive power resource to provide reactive power. In this paper, Wind Power Generation (WPG) and Diesel Generation are considered as typical DG sources when we formulate the model in this paper.

Although there are many advantages to install wind turbines in a distribution system, it has to be noted that wind power generation may result in the reverse power flows from feeder-end nodes to substations, which may cause the voltage variations due to the random-like outputs of wind turbines. The voltage variations may conflict with the standard voltage regulation methods and lead to poor power quality. To solve this problem, appropriate sizes and locations of DGs are suggested to overcome their negative impacts on voltages in [1] and [2]. In [3] and [4], some methods are proposed to determine the introduction limits of DGs. In [5], a real-time voltage regulation method is proposed to regulate the sendingend voltage of a substation node with the given load information and DG locations. In [6], load control is used to regulate the voltage variations introduced by wind turbines. To reduce the negative impacts of wind turbines on voltage profiles, the traditional voltage regulation devices, such as capacitors may not be able to react fast enough to the frequently changed voltages due to the wind power outputs. Static Var Compensators (SVCs) can be used in the distribution systems with wind turbines for fast voltage regulations.

In this paper, we will analysis the impacts of wind turbines on the voltage profiles of distribution systems. To regulate voltages, we propose to use SVCs and diesel generators in distributed systems to provide reactive power support. Both SVCs and generators are fast devices for providing reactive power. They are able to regulate the fast voltage changes due to uncertain wind power outputs. SVCs can control line flows efficiently and regulate voltages continuously. In a distribution system, power quality is one of the main concerns. Although SVCs are more expensive than capacitor banks, it is necessary to install SVCs in a distribution system with wind turbines to overcome the voltage variations and smooth system operation. On the other hand, DGs with traditional technologies, such as, diesel generators, are fast reactive power sources in a distribution system for reactive power support and voltage control [7].

Due to the random-like outputs of wind turbines, the determination of the size and location of a SVC is a critical problem. It is necessary to perform Monte-Carlo simulation to simulate the various outputs of wind turbines. The objectives of reactive power planning are minimizing the cost of system losses, minimizing the installation and operation cost of SVCs and minimizing the reactive power production cost of diesel generators. As SVCs and diesel generators are used for regulating the voltage variations caused by wind turbines, the distribution network companies need to allocate the reactive power support cost among all wind power generators, which are responsible for the cost of installing SVCs. Then, the question is how to allocate the cost. To solve the problem, we will use the Shapely value axiom to allocate the reactive power cost among all the wind power generators. Shapely value criterion is based on the cooperative game theory and with the merit of marginality. The allocation result obtained by Shapely value axiom is accepted by participants for its subsidy-free and equitable manner.

In Section III, we will describe the procedure of using Monte-Carlo simulation to simulate the outputs of wind turbines. In Section IV, the reactive power planning model is formulated for a radial distribution system with DGs. Genetic Algorithm is applied to solve the problem. In Section V, the Shapely value method is used for distributing cost of SVCs among wind power generators. Conclusions are given in section VI.

\section{RANDOM-OUtPUt ASSESSMENT OF Wind POWER GeNERATION BASED ON MONTE-CARLO Simulation OF THE WIND SPEED}

The power production of a wind turbine depends significantly on wind speed, which is an uncertain factor. Monte-Carlo simulation method is a good tool to simulate the probability density function of a random variable like wind speed.

In general, the probability distribution of wind speed is considered following the Rayleigh distribution. The probability density function can be formulated as following,

$$
f(\omega)=\frac{\omega}{a^{2}} e^{-\frac{1}{2}\left(\frac{\omega}{a}\right)^{2}}=2 \frac{\omega}{c^{2}} e^{-\left(\frac{\omega}{c}\right)^{2}}
$$

Where, $\omega$ is the wind speed and $c$ is the scale factor, obtained from historical data.

According to Monte-Carlo simulation method [8], by producing a random number $\gamma$ which satisfies $[0,1]$ uniform distribution, the wind speed can be written as following,

$$
\omega=c \cdot \sqrt{-2 \ln \gamma}
$$

For simplicity, we assume that all wind turbines are located at the same wind farm, so that the correlation coefficients between wind speeds are equal to zeros. For the same wind farm, we can get an independent random wind speed within each time-interval, $\Delta t$ (assuming the wind speed is invariable within $\Delta t$ ). If the simulation cycle is long enough, following large number theorem, the mean value of the statistic data is approximate to the mathematical expectation.

Once the wind speeds at different time-intervals are known, the active power outputs of wind turbines can also be evaluated using Monte-Carlo simulation. Then, the random reactive power outputs can be obtained according to the relationship between reactive power output and active power output of wind turbines [9].

\section{Reactive Power Planning Model}

In this paper, SVCs and diesel generators are used in the 
distribution systems to regulate the voltage fluctuations. An optimal reactive power planning model is applied to determine the optimal sizes and locations of SVC installations and the optimal reactive power outputs of diesel units. The objectives are minimizing installation cost, reactive power operation cost and the cost of system losses. The outputs of wind power generators at each status are included in the power flow constraints to reflect their impacts on distribution systems. The control variables of the optimization model are sizes and locations of SVCs, and reactive power outputs of diesel generators under different wind power output statuses. The binary discrete variables are used to represent whether it is optimal to install a SVC at a node.

\section{A. Objective function}

The objective function is composed of three parts, loss cost, installation cost and production cost.

1) Cost of system loss after reactive power compensation

For a given time-interval, we assume that the load of the distribution system is constant, while the outputs of wind power generators vary with the changes of wind speeds. The cost of system loss $S$ is the sum of the loss costs of all wind power output statuses, as following,

$$
S=K * \sum_{t=1}^{T} T_{t} P_{t}(x)
$$

Where, $P_{t}(x)$ is the active power loss for the wind power output status $t ; T_{t}$ is the duration of status $t ; K$ is energy price.

2) Reactive power support cost of $S V C$

The cost of SVC is usually represented by an approximated linear function with a fixed installation cost and a variable operation cost. The cost of SVC $F$ can be formulated as following,

$$
F=\sum_{i=1}^{I} e_{i}\left(r_{i} Q_{c i}^{0}+c_{i}\right)
$$

Where, $r_{i}$ and $c_{i}$ represent the marginal cost and fixed installation cost of the SVC at node $i$, respectively. The value of binary variable $e_{i}$ depends on whether the SVC is installed ( $e_{i}=1$ ) or not installed $\left(e_{i}=0\right)$ at node $i . Q_{c i}^{0}$ represents the required capacity of the SVC placed at node $i$ to accommodate all wind turbine output statuses.

3) Reactive power production cost of diesel generator

The distributed generators with traditional generation technologies can supply local loads directly without long distance transmission. It is a good option to use distributed generators to control the voltages of distribution systems. In [10], a reactive power cost function for generator has been proposed to represent the operation cost and opportunity cost of providing reactive power. In this paper, we will use a quadratic cost function to simply represent the cost function proposed in [10] for the cost of reactive power provided by diesel generators. The production cost $R$ is formulated as following,

$$
R=\sum_{t=1}^{T} \sum_{i=1}^{I}\left(a_{i}+b_{i} Q_{D G i}^{t}+c_{i} Q_{D G i}^{t}{ }^{2}\right)
$$

Where, $a_{i}, b_{i}$ and $c_{i}$ are the coefficients of the production cost function of the diesel unit at node $i . Q_{D G i}^{t}$ is the reactive power output of the diesel unit at node $i$ under the wind turbine output status $t$.

\section{4) Objective function}

For a distribution system with distributed generations, the objective function of the reactive power planning model is to minimize the sum of the above three cost functions as shown in (1).

$$
\begin{aligned}
\min f=K^{*} \sum_{t=1}^{T} T_{t} P_{t}(x) & +\sum_{i=1}^{I} e_{i}\left(r_{i} Q_{c i}^{0}+c_{i}\right) \\
& +\sum_{t=1}^{T} \sum_{i=1}^{I}\left(a_{i}+b_{i} Q_{D G i}^{t}+c_{i} Q_{D G i}^{t}{ }^{2}\right)
\end{aligned}
$$

\section{B. Constraints}

The constraints are as following,

- Power flow equations

$P_{D G i}^{t}+P_{W P G i}^{t}-P_{L i}=V_{i}^{t} \sum_{j=1}^{I} V_{j}^{t}\left(G_{i j} \cos \delta_{i j}^{t}+B_{i j} \sin \delta_{i j}^{t}\right)$

$Q_{D G i}^{t}+Q_{W P G i}^{t}+Q_{c i}^{t}-Q_{L i}=V_{i}^{t} \sum_{j=1}^{I} V_{j}^{t}\left(G_{i j} \sin \delta_{i j}^{t}-B_{i j} \cos \delta_{i j}^{t}\right)$

- Voltage constraints

$$
V_{i}^{\min } \leq V_{i}^{t} \leq V_{i}^{\max }
$$

- Wind power generator output conditions

$$
P_{W P G i}^{t}=e_{i W P G} P_{W P G i}^{t}, \quad Q_{W P G i}^{t}=e_{i W P G} Q_{W P G i}^{t}
$$

- Diesel generator output conditions

$$
P_{D G i}^{t}=e_{i D G} P_{D G i}^{t}, \quad Q_{D G i}^{t}=e_{i D G} Q_{D G i}^{t}
$$

- Reactive power limits for SVCs

$$
\begin{aligned}
& 0 \leq Q_{c i}^{t} \leq Q_{c i}^{0} \\
& 0 \leq Q_{c i}^{0} \leq Q_{c i}^{\max }
\end{aligned}
$$

- Active and reactive power limits for diesel units

$$
\begin{aligned}
& 0 \leq P_{D G i}^{t} \leq P_{D G i}^{\max } \\
& 0 \leq Q_{D G i}^{t} \leq Q_{D G i}^{\max }
\end{aligned}
$$

\section{Numerical example}

\section{1) Simulation conditions}

A typical 32-node radial distribution system with wind turbines and diesel units is used to test the proposed model. Base on the original distribution system [11], we added three wind power generators at node 17, 24 and 32, respectively. Two diesel units are added at node 10 and 29, respectively. The modified 32 nodes radial distribution system is shown in Fig.1.

Assume the three wind power generators are same and the maximum active power outputs of all generators are $700 \mathrm{~kW}$. They are all located in the same wind farm, which means that 
the random-output characteristics of the three wind power generators are same. So that, Monte-Carlo simulation results of the output of one wind turbine can be used to represent that of the other two wind turbines.

The rated active power and reactive power of the diesel units at node 10 and 29 are $(250 \mathrm{~kW}, 187.5 \mathrm{kVar}$ (lag)) and (50kW, 37.5kVar (lag)), respectively. The voltage limits for all buses are set as [0.9 1.1]. Eight wind power generation statuses are simulated to determine the optimal values of control variables. It is assumed that there are two selectable types of SVC devices, which have capacities of 1000kVar and $1500 \mathrm{kVar}$, respectively.

Assume that the energy price $K=0.05 \$ / \mathrm{kWh}$, the marginal cost and fixed installation cost of a SVC are $3 \$ / \mathrm{kWh}$ and $1000 \$$, respectively. The coefficients of the reactive power production cost functions of diesel generators are identical, and their values are $a_{i}=0.02 \$ / \mathrm{kVar}, b_{i}=0.02 \$ / \mathrm{kVar}$, and $c_{i}=0.01 \$ / \mathrm{kVar}$.

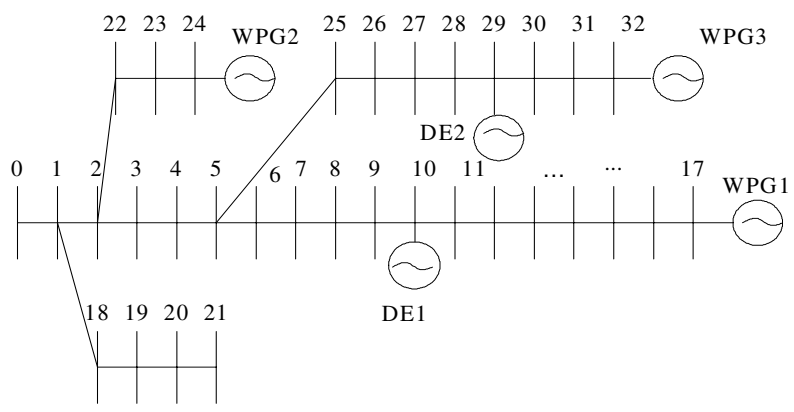

Fig. 1. 32-node distribution system

\section{2) Simulation results}

a) Monte-Carlo simulation

Monte-Carlo simulation has been performed for eight wind speed scenarios. Each scenario is characterized by the mean value of wind speeds and the active and reactive power outputs as shown in Table I.

TABLE I

WIND POWER OUTPUT SIMULATION RESULTS

\begin{tabular}{c|c|c|c}
\hline scenario & $\boldsymbol{C}(\mathbf{m} / \mathbf{s})$ & $\boldsymbol{\omega}(\mathbf{m} / \mathbf{s})$ & $\begin{array}{c}\text { Active and reactive power } \\
\text { outputs }(\mathbf{k W}, \mathbf{k V a r})\end{array}$ \\
\hline 1 & 5.45 & 4.73 & $0-100,-30-0$ \\
\hline 2 & 6.57 & 5.83 & $100,-30$ \\
\hline 3 & 7.25 & 6.57 & $200,-40$ \\
\hline 4 & 8.89 & 8.12 & $300,-60$ \\
\hline 5 & 9.45 & 8.85 & $400,-92$ \\
\hline 6 & 10.54 & 9.73 & $500,-125$ \\
\hline 7 & 11.82 & 11.14 & $600,-145$ \\
\hline 8 & 12.56 & 11.69 & $700,-200$ \\
\hline
\end{tabular}

\section{b) Optimal results of control variables}

In the reactive power optimization procedure, general binary code mechanism would cause excessively long code, which may lead to infeasible solutions and inefficient computations due to the frequent decoding. To overcome the problem and obtain a faster convergence speed and a global convergence rate, based on real and binary encoding mechanisms, Hybrid Encoding Genetic Algorithm (HEGA) [12] has been used to solve the mixed nonlinear programming problem (1)-(8) proposed in this section. Considering all wind turbine output statuses, we use real encoding to represent the outputs of SVCs and diesel generators, and use the binary encoding to express the locations of SVCs easily. Through applying appropriate cross and mutation operators to each encoding mechanism, the results are convergent after 156 iterations. The optimal SVC locations are obtained at node 9, 13, 16 and 30. The optimum sizes for the four SVCs are 1500, 1500, 1000 and 1500 in $\mathrm{kVar}$.

The detailed optimal results under all wind power generation output levels are given in Table II.

TABLE II

DETAILED OPTIMAL RESUlTS UNDER ALl WIND POWER OUTPUT STATUS

\begin{tabular}{|c|c|c|c|}
\hline $\begin{array}{l}\text { Wind } \\
\text { power } \\
\text { output } \\
\text { status }\end{array}$ & $\begin{array}{c}\text { Active and } \\
\text { reactive power } \\
\text { outputs of wind } \\
\text { turbines } \\
(\mathrm{kW}, \mathrm{kVar})\end{array}$ & $\begin{array}{c}\text { Optimal size(kVar) } \\
\text { of SVC @ location } \\
\text { node }\end{array}$ & $\begin{array}{c}\text { Reactive power } \\
\text { outputs of } \\
\text { diesel units } \\
\text { (kVar) @ } \\
\text { node }\end{array}$ \\
\hline 1 & $\begin{array}{l}<100 \\
-30-0\end{array}$ & $\begin{array}{c}1500 @ 9,1500 @ 13, \\
1000 @ 16,1500 @ 30 .\end{array}$ & $\begin{array}{c}300 @ 10 \\
50 @ 29\end{array}$ \\
\hline 2 & $100,-30$ & $\begin{array}{l}\text { 1500@9,1472.8@13, } \\
910.9 @ 16,1500 @ 30 .\end{array}$ & $\begin{array}{c}259.2 @ 10 \\
50 @ 29\end{array}$ \\
\hline 3 & $200,-40$ & $\begin{array}{l}\text { 1500@9,1450.5@13, } \\
818.3 @ 16,1500 @ 30 .\end{array}$ & $\begin{array}{c}300 @ 10 \\
50 @ 29\end{array}$ \\
\hline 4 & $300,-60$ & $\begin{array}{c}\text { 1450@9,1373.1@13, } \\
\text { 866.2@16, } \\
\text { 1420.6@30. } \\
\end{array}$ & $\begin{array}{c}284 @ 10 \\
50 @ 29\end{array}$ \\
\hline 5 & $400,-92$ & $\begin{array}{c}1400 @ 9,1337.4 @ 13, \\
810.3 @ 16, \\
1386.5 @ 30 .\end{array}$ & $\begin{array}{c}300 @ 10 \\
50 @ 29\end{array}$ \\
\hline 6 & $500,-125$ & $\begin{array}{c}1340.5 @ 9, \\
1286.5 @ 13, \\
739.1 @ 16, \\
1260.7 @ 30 . \\
\end{array}$ & $\begin{array}{c}300 @ 10 \\
50 @ 29\end{array}$ \\
\hline 7 & $600,-145$ & $\begin{array}{l}\text { 1250@9,1200@13, } \\
700 @ 16,1200 @ 30 .\end{array}$ & $\begin{array}{c}300 @ 10 \\
50 @ 29\end{array}$ \\
\hline 8 & $700,-200$ & $\begin{array}{c}1144.9 @ 9, \\
1157.5 @ 13, \\
710.5 @ 16, \\
1160.3 @ 30 .\end{array}$ & $\begin{array}{c}300 @ 10 \\
50 @ 29\end{array}$ \\
\hline
\end{tabular}

From Table I, we can see that wind power generators generate active power and absorb reactive power at the same time. The cost of using wind energy is that reactive power must be supported from distribution systems to compensate the voltage variations caused by wind turbines. Moreover, the reactive power compensation devices should be able to regulate voltage continuously. This means that, both the advantages and the disadvantages should be considered when introducing DGs to distribution systems.

The power flow solution at each WPG output profile has been analyzed. By comparing the outputs of SVC devices at all time-intervals in Table II, it is found that when the active power output of each wind power generator increases, the reactive power requirement decreases. The reason is that distribution lines have large $R / X$ ratio. When the active power outputs of WPGs at feeder-end nodes increase, they may 
cause reverse power flows and raise system voltages. Although a wind turbine consumes reactive power when generating active power, the positive impact of raising voltage levels by increased active power output is greater than the negative impact of decreasing voltage levels by increased reactive power consumptions of WPGs. This provides a good signal to decision makers to introduce the appropriate sizes of DGs to supply electric energy while having positive effect of raising system voltages to a certain extent.

In Table III, we list the total system losses and the system lowest voltage levels of one wind scenario with and without SVC installation. It is found that the losses are reduced while the voltage level is raised significantly by installing SVCs. Fig. 2 indicates the probabilistic voltage level at node 5 under different SVC outputs. In the figure, the voltage level refers to the difference between actual voltage level and reference voltage level. It is found that a certain amount capacities of SVCs can help to eliminate voltage deviations significantly.

TABLE III

SySTEM LOSSES AND LOWEST VolTAGE LEVELS WiTH AND WiTHOUT SVC

\begin{tabular}{c|c|c}
\hline Case & $\begin{array}{c}\text { System losses } \\
(\mathbf{k W})\end{array}$ & $\begin{array}{c}\text { Lowest system } \\
\text { voltage level }(\mathbf{p u})\end{array}$ \\
\hline With SVC & 630 & 0.90 \\
\hline Without SVC & 3150 & 0.59 \\
\hline
\end{tabular}

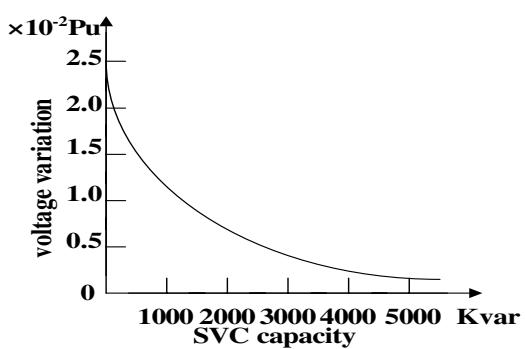

Fig. 2. voltage variation at node 5

\section{Allocation of Reactive Power Support Costs of SVCS}

The minimum cost has been obtained to install SVCs in a radial distribution system in Section IV. The main purpose of installing SVCs is to regulate the voltage variations caused by wind turbines. In this section, we will discuss who should pay for the reactive power support cost, distribution companies, consumers or wind power generators? The next question is how to allocate the cost? Since the cost of installing SVCs is caused by the random-outputs of wind turbines, we propose to allocate the cost among wind turbines in the distribution system. Then, the question is how to allocate the cost?

In this paper, we propose to use Shapley value criterion in the cooperative theory to allocate the cost. The Shapley value criterion can provide an equitable and efficient cost allocation. The game-theoretic Shapley value has been applied successfully in many public cost allocation problems for its desirable properties of coalition monotonicity and the concept of "stable solution". In addition, it is the only one that satisfies four economic equality axioms as below,

- Efficiency: the total cost is covered completely by agents;

- Anonymity: the Shapley value commutes with the permutations of agents;

- Additivity: the allocation result of a problem is the sum of that of sub-problems;

- Dummy: an agent with zero marginal contribution will be charged with zero.

Shapley value is a cost allocation method with many virtues. We apply it in this paper to allocate reactive power costs of SVCs among wind power generators.

\section{A. Application of Shapley value axiom to the cost allocation of SVC}

The Shapley Value allocation can be expressed as following [13]:

$$
X_{i}=\sum_{s} \frac{(|s|-1) !(n-|s|) !}{n !} *[V(s)-V(s-\{i\})]
$$

Where, $X_{i}$ is the allocated cost to agent $i . s$ represents a subcoalition including agent $i . n$ denotes the total number of agents. $V(s)$ is the characteristics function, i.e., the cost associated with coalition $s$, and $V(s-\{i\})$ is the cost after dropping designated agent $i$.

Note that the coefficient of $[V(S)-V(s-\{i\})]$ represents the number of coalitions of size $s$ containing the designated agent $i$. According to (9), each agent $i$ is allotted a value equal to its expected marginal contribution across all possible coalitions, which is fair and desirable for each agent in the game.

For the numerical example in section IV, the reactive power planning model can be used to obtain the reactive power requirement of SVCs for various combinations of wind power generators. The optimal results under each WPG coalition are shown in Table IV. In the table, number 1, 2 and 3 represent the wind power turbines at node 17, 24 and 32, respectively.

TABLE IV

OPTIMAL RESULTS UNDER ALL WPG COALITIONS

\begin{tabular}{c|c}
\hline WPG coalition & $\begin{array}{c}\text { Optimal size (kVar) of SVC @ location } \\
\text { node }\end{array}$ \\
\hline \multirow{2}{*}{1} & $1400 @ 9,1500 @ 13$, \\
& $1000 @ 16,1500 @ 30$. \\
\hline \multirow{2}{*}{2} & $1500 @ 9,1500 @ 13$, \\
& $1000 @ 16,1500 @ 30,600 @ 23$. \\
\hline \multirow{2}{*}{3} & $1600 @ 9,1500 @ 13$, \\
& $1000 @ 16,1500 @ 30$. \\
\hline \multirow{2}{*}{1,2} & $1400 @ 9,1500 @ 13$, \\
& $1000 @ 16,1500 @ 30$ \\
\hline \multirow{2}{*}{1,3} & $1400 @ 9,1500 @ 13$, \\
& $1000 @ 16,1400 @ 30$. \\
\hline \multirow{2}{*}{2,3} & $1500 @ 9,1500 @ 13$, \\
\hline \multirow{2}{*}{$1,2,3$} & $1000 @ 16,1400 @ 30,600 @ 23$. \\
\hline
\end{tabular}


According to the SVC cost function and related cost data in section IV, all coalition costs $V(s)$ are calculated as following,

$$
\begin{aligned}
& V(1)=15700 \$ ; \quad V(2)=18800 \$ \quad V(3)=16300 \$ ; \\
& V(12)=15700 \$ ; \quad V(13)=15400 \$ ; \\
& V(23)=18500 \$ ; \\
& V(123)=16000 \$ .
\end{aligned}
$$

Note that $V(0)=0$.

Assume $X_{1}, X_{2}$, and $X_{3}$ are the reactive power support costs allocated to wind power generators at bus 17, 24 and 32 respectively. In accordance with Shapley value formulation, the Shapley values can be found as,

$X_{1}=3733.3 \$, X_{2}=6833.3 \$$, and $X_{3}=5433.3 \$$.

The results of $X_{1}, X_{2}$, and $X_{3}$ are the allocation results of the reactive power cost of SVCs to three wind power generators. Assuming each capacity cost has a 10-year lifetime, the distribution company can charge a fixed reactive power support cost of $1.02 \$ /$ day to the WPG at bus $17,1.87 \$ /$ day to the WPG at bus 24, and 1.49\$/day to the WPG at bus 32 .

\section{B. Result analysis}

Shapely value axiom determines the reactive power support cost allocation among wind power generators in a distribution system. The potential benefits of using this criterion are,

- The first merit of Shapley value axiom is the property of marginality, which means that the marginal contribution of a participant is the only factor that can decide the allocation. As shown in table IV, the wind turbine at node 24 consumes more reactive power than other wind turbines do. So that this wind turbine should be charged for a higher capacity fee. The result is equitable and subsidy-free.

- The result is efficient, and can satisfy both individual and coalitional rationality, for

$$
\begin{aligned}
& X_{1}+X_{2}+X_{3}=V(123) \\
& X_{1} \leq V(1), X_{2} \leq V(2), X_{3} \leq V(3) \\
& X_{1}+X_{2} \leq V(12), X_{1}+X_{3} \leq V(13), \\
& X_{2}+X_{3} \leq V(23)
\end{aligned}
$$

- It provides some economic signals to decision-makers to determine the appropriate locations of DGs. For example, bus 24 is not a good option to build a wind turbine due to the high reactive power support cost might be caused by the wind turbine at this bus.

- If the number of coalitions is not a big number, Shapely value calculation is always feasible, and the solution is unique and stable.

\section{CONCLUSIONS}

In this paper, a reactive power planning model has been proposed for a radial distribution system with wind turbines. SVCs and DG sources with traditional technologies are used for reactive power support. The optimal solutions are calculated to determine the optimal sizes and locations of SVC installations. The costs of reactive power support due to the introduction of wind turbines are allocated using Shapley value axiom. The result is acceptable to all participants and the solution has been proved unique and stable.

\section{REFERENCES}

[1] Z. Wang, S. Zhu, "Impacts of DG to system voltage", Electric power system and its automation.Vol.28, No.16, Aug, 2004.

[2] Ljubomir Kojovic and Franksville WI, "Impact of DG on Voltage regulation”, 2002 IEEE Power Engineering Society Summer Meeting, Volume 1, 2002.

[3] J.E.Kim et al. "Methods of determining the introduction limit of dispersed generation systems in a distribution system from the viewpoint of voltage regulation", IEE Japan Trans. Vol.16, No.12, 1996.

[4] J.E.Kim et al. "Impacts of Dispersed storage and generation on the sending-end voltage in a distribution substation and a method of their introduction limits", Proc. of ICEE, July, 1995.

[5] J.H.Choi, J.C.Kim, "Advanced voltage regulation method at the power distribution systems Interconnected with dispersed storage and generation systems", IEEE Trans. on Power Delivery, Vol.15, April 2000.

[6] N. Sott, D. J. Atkin son and J. E. Morrel, "Use of load control to regulated voltage on Distribution networks with embedded generation" IEEE Trans on power systems. Vol.17, No, 2, may 2002.

[7] Y. He, Z. Wen. Electric power system analysis, HuaZhong university of science and technology press.

[8] A.E.Feijoo, J.Cidras and J.L.G.Dornelas, "Wind speed simulation in wind farms for steady-state security assessment of electrical power systems" IEEE Transactions on Energy Conversion, Vol.14, No.4, December 1999.

[9] European Wind Turbine Testing Procedure Developments. DDSI Danka Services International, 2001.

[10] J. Zhong, K. Bhattacharya, "Toward a competitive market for reactive power" IEEE Transactions on Power Systems, Vol 17, No 4, Nov 2002.

[11] M.E.Baran and F.F.Wu, "Network Reconfiguration in distribution systems for loss reduction and load balancing" IEEE Trans. on power Delivery, Vol.4, No.2, Apr. 1989.

[12] L. Yang, X. Yang and Y. Liu, "Evolutionary computation with adaptive hybrid binary and real encoding method", Computer Engineering, Vol.28, No.11, 2002.

[13] H. Moulin. Axioms of cooperative decision making, Cambridge university press.1988.

[14] M.E. Baran and F.F. Wu, "Optimal Capacitor Placement on Radial Distribution Systems" IEEE Trans. On Power Delivery, Vol.4, No.1, pp.725-734,Jan.1989.

[15] G.Carpinelli, G.Gelli, F.Pilo and A.Russo, "Distributed generation siting and sizing under uncertainty", 2001 IEEE Porto Power Tech conference.

[16] D. Chattopadhyay, B.B. Chakrabarti, "Reactive power planning incorporating voltage stability", International Journal of Electrical Power and Energy Systems, vol. 24, 2002, pp. 185-200.

\section{BIOGRAPHIES}

Lin Chen received the B. Sc. (Eng.) degree from Chang Sha University of Electric Power, Changsha, China, in 2002. Currently, she is a Ph.D. candidate at Zhejiang University, China. Her major research interests are distributed generation and electricity market.

Jin Zhong (S'00, M'05) received the B. Sc. (Eng.) degree from Tsinghua University, Beijing, China, in 1995 and the M. Sc. (Eng.) degree from the Electric Power Research Institute, Beijing, in 1998, and the Ph.D degree from Chalmers University of Technology, Gothenburg, Sweden, in 2003. She is an Assistant Professor in the Department of Electrical and Electronic Engineering at the University of Hong Kong.

Deqiang Gan received the Ph.D. in Electrical Engineering from Xian Jiaotong University, Xian, China, in 1994. Currently, he is with Zhejiang University, China. He was a Senior Analyst in ISO New England, Inc. where he worked on issues related to the design, implementation, and economic analysis of electricity markets. Prior to joining ISO New England, Inc., he held research positions at several universities in USA and Japan. 